PACS 03.75.Kk, 67.85.-d

\title{
Pressure-induced vortex rings multiplication as a source of vorticity in superfluids ${ }^{1}$
}

\begin{abstract}
I.A. Pshenichnyuk
i.pshenichnyuk@skoltech.ru

Center for Photonics and Quantum Materials, Skolkovo Institute of Science and Technology, Skolkovo Innovation Center, Building 5, Moscow 143026, Russia.

A modified version of the Gross-Pitaevskii equation (GPE) combined with the Newton equation is used to model the propagation of a heavy particle through a quantum fluid. In the considered process, the particle, moving with an initial velocity, creates a vortex ring excitation in the fluid. A subsequent change of pressure, which appears because of the interaction of the particle with a sound wave, initiates the vortex multiplication process. As a result of the following vortex interplay, the moving particle becomes surrounded by a decaying turbulent cloud with high degree of vorticity. It is demonstrated how at certain stage of evolution of the cloud, the kinetic energy of the fluid circulation around the particle increases in a resonant manner. In accordance with the hydrodynamic Bernoulli principle, an increase of the speed of the fluid (kinetic energy) results in a decrease of pressure (potential energy) around the particle. Such mechanism may help to understand the details of experiments with exploding electron bubbles in liquid helium, as a result of the interaction with sound waves.
\end{abstract}

Keywords: quantum vortex, quantum material, turbulence, superfluidity.

\section{Introduction}

One of the peculiar properties of superfluids (like liquid helium-4 below the lambda point or trapped ultracold atoms) is the quantization of velocity circulation, which leads to the existence of quantum vortex filaments. Along with superfluidity, understanding of this phenomenon is not only a question of fundamental science, but also an important milestone in modeling and investigation of novel quantum materials. It is well known that in a bucket with superfluid helium, being rotated with a sufficient angular velocity, an organized array of quantum vortices is formed. Each vortex curries a quantum of velocity circulation $h / m$ (where $h$ is the Planck constant and $m$ is a mass of a helium atom) and appears in a node of an imaginary triangular lattice, also known as a vortex lattice [1]. Quantum vortices are stable topological defects. Being nucleated, by one way or another, they can move in a fluid and interact with each other through vortex reconnection events [2]. Under certain conditions, vortex filaments may get tangled, producing a disordered state known as a quantum turbulence. It may happen, for example, as a result of sudden spin-down in a rotating bucket experiment [3]. Quantum vortices may interact with electrons, atoms and other impurities, capturing them [4]. Filaments with sufficient density of captured particles become experimentally "visible" and can be photographed [5]. Such doping is a main practical mechanism of vortex lines visualization, the task that is challenging otherwise because of a small size of a vortex core (usually of the order of few angstroms). Having in mind the listed features one may conclude, that the presence of quantum vortices may result in an unusual form of internal structure organization of a matter, which, in turn, allows to expect unusual macroscopic properties. Recently, vortex lattices were discovered experimentally in helium nanodroplets, produced during the expansion of a liquid helium into a vacuum [6]. Bragg patterns, revealing the existence of periodic vortex structures, were found using the femtosecond $\mathrm{x}$-ray diffractive imaging on Xe-doped helium droplets. These experiments demonstrate, that the presence of quantum vortices is responsible for the unusual geometry of droplets and for the increased droplets stability, which gives an example of a macroscopic manifestation of vortices. Theoretical works, justifying the observed results follow $[7,8]$.

There are different ways to create vorticity in superfluids. Along with the mentioned above method of rotating bucket, which creates an array of linear vortices, it is known, that a moving spherical object (like a particle, or macroscopic cluster) can nucleate vortex rings [9]. While linear vortices may terminate only near boundaries of the fluid, rings may occupy any limited part of the volume (depending on their radius), being in this sense more geometrically compact excitations. It is worth mentioning that the spectrum of elementary excitations in quantum fluids includes different types of objects, like, for example, rarefaction pulses, with lower excitation energy than vortex rings. A particle, thus, should possess enough energy to be able to nucleate a ring.

1 This paper is dedicated to my father, who passed away. 
Depending on the initial kinetic energy, the ring may detach itself from the particle or propagate together with it.

One more property of quantum vortices, which is important for the subsequent discussion of the results, reveals itself when we change the pressure in the system. As was demonstrated in [10], the decrease of pressure results in the vortex core expansion. When the pressure is back to normal vortex shrinks (the contraction phase) and, at a certain moment, may split, producing few more filaments. The vortex ring multiplication phenomenon was investigated at different temperatures in the recent work [11].

Both properties listed above, namely the nucleation of a vortex ring by a moving particle and the vortex ring multiplication under the influence of pressure take place during the interaction of electrons, propagating in liquid helium, with sound waves. Such experiments were performed by the group of H.J. Maris [12,13].

They investigated the stability of electron bubbles (an electron, being placed into the liquid helium, forms a soft bubble with a radius of about $15 \AA$ ) subject to pressure variations. It was demonstrated, that at certain critical value of pressure, which is defined by an amplitude of sound waves, electron bubbles become unstable and explode. In addition, it was shown, that electron bubbles trapped by vortex lines explode at a different pressure than free bubbles. It is explained by an increased circulation of the superfluid around a bubble, which is caused by the presence of a vortex. According to the Bernoulli principle, such circulation decreases the pressure around the bubble, which makes it less stable. Along with bubbles and trapped bubbles, Maris and his colleagues report the existence of another type of objects, which they call an unidentified electron object (UEO), with even lower explosion threshold. One possible explanation of the existence of such objects is connected with the presence of few vortices in a close vicinity of an electron. Taking into account a low probability of such a coincidence, the exact mechanism of appearance of UEO remains unclear.

In the present work, we introduce the results of modeling of a particle moving through a quantum fluid. It is shown how a vortex ring is nucleated by the particle and, further, how the pressure variation initiates the vortex expansion, which is followed by the contraction and subsequent multiplication of the ring. As a result, few vortex rings appear in a close vicinity of the particle. Such a mechanism may explain the existence of UEO in the works of Maris et al. The Bernoulli instability effect is evaluated quantitatively by calculating the kinetic energy of the fluid circulation around the particle as a function of time. It is demonstrated how the circulation reaches its peak value at a certain stage of evolution of the system. The results of modeling are presented in the section 3 , immediately after the discussion of the used theoretical formalism in the section 2. The conclusion is given in the section 4 .

\section{Theory}

The modified version of the Gross-Pitaevskii equation (also known in mathematics as nonlinear Schrödinger equation) is used to model the quantum fluid [11]. GPE-approach is known to give good qualitative results for quantum fluids and Bose condensates with weak interaction between the particles. It was also shown that the Landau two fluid model of liquid helium could be derived from GPE with rather general form of nonlinearity (for the discussion see $[14,15]$ ). In the sense of mathematical equivalence to the model of Landau, GPE can be used to describe the helium with strongly correlated particles (in addition to that, GPE formalism, obviously, gives an adequate self-consistent description of quantum vortices dynamics). GPE approach allows to incorporate different types of modifications which makes it quite flexible for the description of various properties of fluids. Nonlocality of interactions between the particles, for example, can be taken into account explicitly, which allows to introduce the roton minimum in the dispersion curve for helium [16]. The correct equation of state of the fluid can be built-in into GPE to study pressure variation effects [11]. GPE equation was also successfully used to describe quantum fluids at finite temperatures [17].

In the present work we use GPE with the nonlinearity of the 7-th order (versus the 3-rd order nonlinearity in the original GPE), which appears when we consider the correct equation of state for the liquid helium (see [11] for the details). For the practical computations GPE is usually taken in the dimensionless form, which reads

$-2 i \frac{\partial \psi}{\partial t}=\left[\nabla^{2}+U+|\psi|^{2}+\chi_{4} \gamma|\psi|^{4}-\chi_{6} \gamma^{2}|\psi|^{6}+\mu\right] \psi$

The distance here is assumed to be in healing lengths $\xi=1 \AA$, time in $\xi^{2} m / \hbar$ and the order parameter $\psi$ is scaled by the factor $\psi_{\mathrm{a}}=0.147833 \AA^{-3 / 2}$, which represents the uniform unperturbed state of helium. It is chosen to reproduce the known value of the liquid helium density at atmospheric pressure. Parameters $\chi_{4}=1.10337$ and $\chi_{6}=1.64744$ are chosen to fit the correct equation of state of the fluid. The chemical potentials is taken in the form $\mu=\chi_{6} \gamma^{2}-\chi_{4} \gamma-1$. The parameter $\gamma$ is introduced in the model to control the pressure [11]. In the presented calculations it changes between 0.96 and 1.04 , which corresponds to the variation of pressure by \pm 1.46 bar ( $\gamma=1$ corresponds to the atmospheric pressure). Spherically symmetric external potential $U$ is created by the moving particle. It is taken in the form

$$
U\left(\boldsymbol{r}, \boldsymbol{r}_{0}\right)=\frac{1}{1+e^{\frac{\left|r-r_{0}\right|-r_{e}}{\sigma}}},
$$

where $\boldsymbol{r}_{0}$ - is the current position of the particle, $r_{\mathrm{e}}=5$ and $\sigma=0.8$.

To describe the behavior of the particle the classical Newton equation is used [9]

$$
M \ddot{\boldsymbol{r}}_{0}=\boldsymbol{F}+\int d^{3} r \frac{d U}{d \boldsymbol{r}}|\psi(\boldsymbol{r}, t)|^{2},
$$

where $M$ is the mass of the particle and $\boldsymbol{F}$ is the external force. It can be created, for example, by the electric field, which was used in the experiment [12] to gain more control over the particles. The second term in the right hand side of the equation 3 represents the influence of the fluid on the particle.

Similar to the work [9] we consider the movement of heavy particles. In the present simulation $M=20000$ is assumed, which corresponds to the mass of a cluster, which contains 
13 atoms of Xe. As it was described above, electrons in liquid helium form bubbles, which explode under the influence of pressure jumps. Such explosions make the analysis of superfluid circulation around them too complicated. In this paper, we focus on the generation of vorticity initiated by the vortex ring multiplication. For this purpose, it is more convenient to consider heavy, electrically neutral particles with the minimized bubble effect and no explosions involved.

The system of equations 1 and 3 is solved numerically in a 3D domain with dimensions $100 \times 150 \times 100 \AA$ and periodic boundary conditions. To discretize the domain $200 \times 300 \times 200$ grid points were used. The 4 -th order Runge-Kutta algorithm is applied to calculate the propagation of the system in time.

\section{Results and discussion}

The particle in our model starts to move through the liquid with an initial kinetic energy $E_{0}=31.2 \mathrm{meV}$. With such an energy deposit it moves fast enough to nucleate a vortex ring, which is completely formed at the moment of time $t=50 p s$. The ring in our simulation does not detach from the particle, but propagates together with it. Such a composite object is shown on Fig. 1, where we plot the cross-section of the condensate density $|\psi(x, y, z=0)|^{2}$ at the moment of time $t=65 p s$ after the beginning of the simulation. Both the particle and the vortex can be seen on the figure as a minima of the condensate density. In the first case, the minimum appears because of the displacement of the condensate by the potential of the particle. In the second case, the vanishing liquid density inside the vortex core is a fundamental property of the vortex (the same is true for classical vortices in gases and liquids). These facts allow us to use density plots for the visualization of two types of objects with different nature. Arrows on Fig. 1 show the velocity circulation field, which helps to identify the vortex ring.

When the vortex ring is nucleated at $t=71 p s$, we reduce the pressure by 1.46 bar. The vortex ring starts to grow in size, denoting the beginning of the expansion phase, which lasts for 63ps. After that, we increase the pressure by 2.92 bar to initiate the contraction phase. According to the experiment [12], pressure variation $\Delta p= \pm 1.46$ bar is too small to cause an electron bubble destabilization. As was already stated above, in this paper we consider the regime with no explosions. At the same time, the pressure variation used here is enough

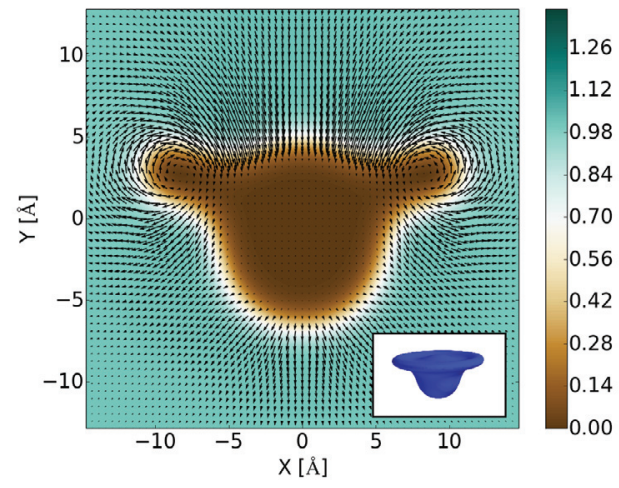

Fig. 1. (Color online) The $2 \mathrm{D}$ cross-section of the superfluid density $\rho=|\psi(x, y, z=0)|^{2}$ at $t=65 p s$. The fluid velocity circulation field is plotted with arrows. The 3D isosurface is shown in the inset. to cause resonant increase in the circulation of the fluid around the particle. The details of the vortex expansion and contraction are shown on Fig. 2, where we plot the isosurface $|\psi(x, y, z)|=0.35$. As in the $2 \mathrm{D}$ example above, it allows to visualize both the particle and quantum vorticity. The process of expansion, contraction and multiplication of a vortex, produced by a moving particle, is cylindrically symmetric. To brake this symmetry and, thus, consider more experimentally realistic situation we add a small side projection to the particle velocity when the ring is already formed. It is clearly seen on the picture how the vortex ring splits during the contraction phase, producing at least two rings and additional turbulent structures, which appear in a close vicinity of the particle (see Fig. 3). It is worth mentioning that in general, without the vortex multiplication, such a situation is rare, because few vortices should approach the particle at the same time to a distance of few angstroms. Appearance of additional vortices near the particle causes the growth of fluid circulation and, with respect to the Bernoulli principle, the decrease of pressure. Such mechanism can easily destabilize an electron bubble, decreasing its explosion threshold. It can explain the existence of UEO, which explode at lower pressure than ordinary electron bubbles and even bubbles, which are trapped by single vortices [12].

To evaluate the Bernoulli destabilization effect quantitatively we compute the kinetic energy of the fluid circulation in a $3.2 \AA$ thick layer $\Gamma$ around the particle as a function of time

$$
E=\frac{1}{8 m} \int_{\Gamma} \frac{\left|\psi \nabla \psi^{\dagger}-\psi^{\dagger} \nabla \psi\right|^{2}}{\psi \psi^{\dagger}} d x d y d z
$$

This quantity is shown on Fig. 4. Vertical dashed lines denote the moments of time where pressure jumps occur. At the beginning of the simulation, the circulation energy is equal to zero. Then it grows smoothly, corresponding to the nucleation of the ring, which is completely formed in 50ps. After the beginning of the expansion phase, the energy goes down. It happens because the vortex ring grows and its core moves away from the particle. At the certain moment of time after the increase of pressure $(t=175 p s)$ the circulation energy rapidly grows producing a resonant-like peak. The maximum value of circulation during the resonance is about 10 times larger than the circulation produced by a single vortex ring. It is interesting that this peak does not appear immediately after

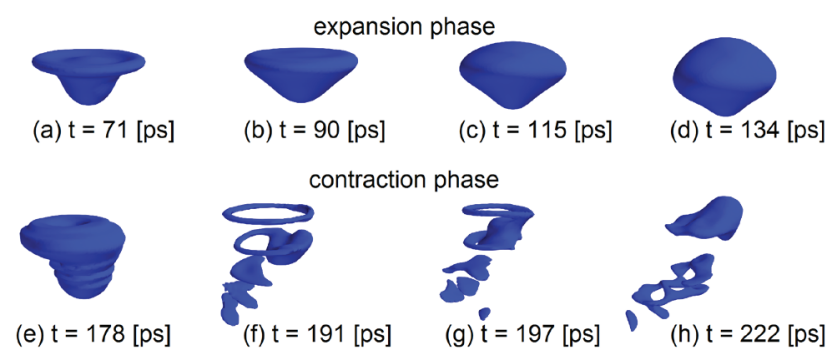

Fig. 2. The evolution of the turbulent cloud is visualized using a superfluid density isosurface $|\psi(x, y, z)|=0.35$. It is shown how the vortex ring expands (a)-(d), then shrinks and multiplies (e)-(g). The decay of the turbulent state is shown at (h). 


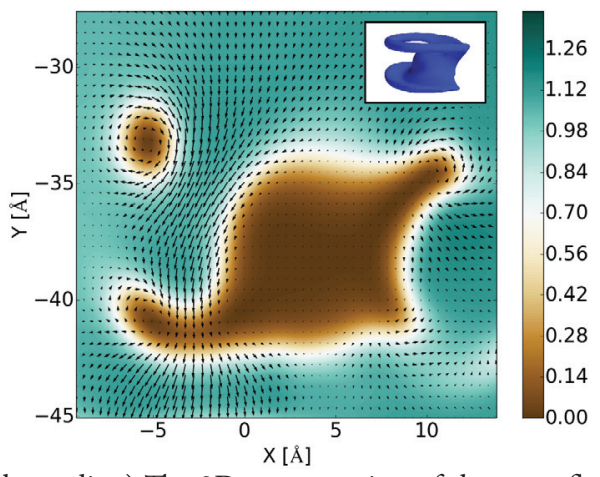

Fig. 3. (Color online) The $2 \mathrm{D}$ cross-section of the superfluid density $\rho=|\psi(x, y, z=0)|^{2}$ at $t=197 p$ s. The fluid velocity circulation field is plotted with arrows. The $3 \mathrm{D}$ isosurface is shown in the inset.

the increase of pressure, but 50ps later. When the system is located at the point 1 (see Fig.4), short before the resonance, the vortex evolution switches from the contraction phase to the multiplication phase. At the point 2, where the energy is maximal, we can observe the state with few vortex rings near the particle. At the point 3, immediately after the resonance, the turbulent state decays. It is interesting to mention that, after the decay, circulation energy does not go to zero but reaches the value close to the one produced by a single vortex ring before the expansion.

The turbulent state, which is responsible for the appearance of the circulation energy peak, is not stable and decays in approximately 100ps after the decrease of pressure. In the considered process, we do not observe the situation when the particle is trapped by few vortex rings, which appear after the multiplication. It is rather a decaying quantum turbulence produced by the scattering of sound waves on moving particles. Despite the short relaxation time, such phenomenon seems to be quite common in quantum fluids. Each particle, assuming it possesses enough energy, may pass through the described phases an unlimited number of times. In some volume of quantum fluid filled with moving impurities subject to pressure variations, there always exists a statistical fraction of particles surrounded by turbulent clouds.

There is one more fact, which supports the turbulent nature of UEO. In the experiment [12] unidentified objects were detected only at low temperatures, while ordinary electron bubbles and trapped bubbles appeared in the whole range of temperatures. In the work [11] it is shown, that the ring multiplication effects is suppressed at high temperatures. It confirms the connection between the multiplication phenomenon and high instability of electron bubbles. The results discussed here do not contradict another possible mechanism, wherein long living UEO are produced by impurities trapped by few vortices, which appear as a result of multiplication. The search of such objects may be the topic of future works.

\section{Conclusions}

The interaction of sound waves with particles, propagating in a superfluid, may result in appearance of decaying turbulent clouds around the particles. Turbulence emerges because of

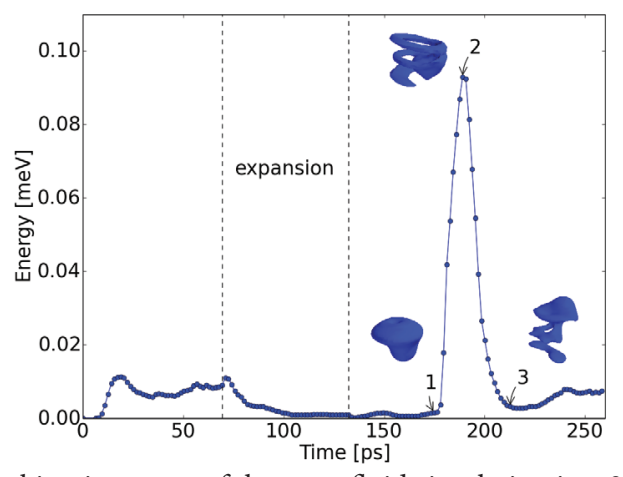

Fig. 4. The kinetic energy of the superfluid circulation in a $3.2 \AA$ thick layer around the particle as a function of time. The dashed lines mark the pressure changes.

pressure-induced multiplication of vortex rings, produced by particles. Each particle may pass through the turbulent phase many times. At a certain stage of evolution of the cloud, vorticity around the particle grows in a resonant manner. One of the possible manifestations of this effect is the appearance of UEO in the experiments with exploding electron bubbles.

Acknowledgments. I would like to thank my supervisor, Prof. Natalia Berloff, for the guidance and numerous discussions. Without her support, this paper would never be written. This work was financially supported by Ministry of Education and Science of Russian Federation \# 1425320 (Project DOI: RFMEFI58114X0006).

\section{References}

1. E.J. Yarmchuk, M.J.V. Gordon, R. E. Packard. Phys. Rev. Lett. 43, 214 (1979).

2. J. Koplik, H. Levine. Physical Review Letters 71, 1375 (1993).

3. P.M. Walmsley, A.I. Golov, H.E. Hall, A.A. Levchenko, W.F. Vinen. Phys. Rev. Lett. 99, 265302 (2007).

4. N.G. Berloff, P.H. Roberts. Phys. Rev. B 63, 024510 (2000).

5. G.P. Bewley, D.P. Lathrop, K.R. Sreenivasan. Nature 441, 588 (2006).

6. L.F. Gomez, K.R. Ferguson, J.P. Cryan, C. Bacellar, R.M.P. Tanyag, C. Jones et al. Science 345, 906 (2014).

7. F. Ancilotto, M. Pi, M. Barranco. Phys. Rev. B 90, 174512 (2014).

8. F. Ancilotto, M. Pi, M. Barranco. Phys. Rev. B 91, 100503 (2015).

9. T. Winiecki, C.S. Adams. Europhys. Lett. 52, 257 (2000).

10. N.G. Berloff. Fluid Dyn. Res. 41, 051403 (2009).

11. N.G. Berloff, M. Brachet, N.P. Proukakis. Proc. Natl. Acad. Sci. 111, 4675 (2014).

12. A. Ghosh, H.J. Maris. Phys. Rev. Lett. 95, 265301 (2005).

13. J. Classen, C.K. Su, H.J. Maris. Phys. Rev. Lett. 77, 2006 (1996).

14. J.A. Geurst. Phys. Rev. B 22 (7), 3207 (1980).

15. C. Coste. European Physics Journal B 1 (2), 245 (1998).

16. N.G. Berloff. J. Low Temp. Phys. 116 (5), 359 (1999).

17. N.G. Berloff, A.J. Youd. Phys. Rev. Lett. 99 (14), 145301 (2007). 